\title{
Blockchain-based Fog-Enabled Energy IoT Architecture
}

\author{
You-jin Song ${ }^{1}$ and Jae-Kyu Lee ${ }^{2}$ \\ ${ }^{1}$ Dept. Information Management, Dongguk University at Gyeongju Campus, Korea \\ ${ }^{2}$ Dept. Techno-Management Cooperation Course, Dongguk University at Gyeongju \\ Campus, S. Korea \\ ${ }^{1}$ song@dongguk.ac.kr, ${ }^{2}$ jaekyulee@dongguk.ac.kr
}

\begin{abstract}
Recently, the paradigm of the power industry has been digitizing around renewable energy. While this shift is accelerating towards energy-information and communications technologies (ICT) convergence, there will be many changes in energy policy in the future. In particular, in terms of energy demand management, locally centered decentralization will take place, and microgrid-type virtual power plants will be built on a regional basis according to the activation of distributed energy resources (DER) including renewable energy. To this end, business models such as electricity trading aggregation should also be considered. In this paper, we design an energy blockchain transaction architecture that combines blockchain and the energy Internet of Things (IoT) in a virtual power plant environment. The system presented in this paper can be integrated with a control system that requires DER and intermediary trading through an intermediary using web-based software. In addition, it is possible to promote the activation of energy brokerage transactions by preparing an environment for efficient trading by collecting a large amount of advanced metering infrastructure (AMI) information in a fog server.
\end{abstract}

Keywords: Energy IoT, Blockchain, Smart contract, Energy cloud, Fog computing

\section{Introduction}

The era of the third industrial revolution, represented by Internet digitalization and characterized by information and communications technologies (ICT) and automation, is shifting to the fourth industrial revolution through a paradigm shift that breaks down the boundaries between industries through intelligentization and hyper-connectivity. Big data, the cloud, artificial intelligence, and mobile and Internet of Things (IoT) technologies, which are innovations that triggered the fourth commercial revolution, are fused with energy technologies, creating changes in the industrial structure and new added values.

Traditional energy systems, which were integrated and centrally supplied through largescale installations, were operated to create value through the simple sale of a single energy source. However, with the development of small-scale facility technologies such as energy storage devices and the application of ICT, the increase in distributed power supplies and the bi-directional data exchange on the supply-demand side have enabled the operation of energy systems digitized around renewable energy. As the transition to energy-ICT convergence accelerates, there will be many changes in future energy trade demand policies [1].

Article history:

Received (November 2, 2019), Review Result (December 11, 2019), Accepted (January 19, 2020) 
In this way, a combination of technological advances and competitive market structures is emerging as a new energy management system that creates value through the expansion of small-scale distributed power supplies and various energy service combinations. In particular, in terms of energy demand management, locally centered decentralization will take place, and microgrid-type virtual power plants (VPPs) will be built on a regional basis according to the activation of distributed energy resources (DER) including renewable energy. Business models such as aggregation will also need to be reviewed [2].

On the other hand, the era of the third industrial revolution, represented by the Internet and digitalization, and characterized by ICT and automation, is a paradigm that broke down the boundaries between industries through intelligence and hyper-connectedness. It is changing to the era of the fourth industrial revolution. In particular, IoT technology connects the physical things in the real world and virtual things in the cyber environment through the Internet. It is the Internet infrastructure of the future that enables people to provide various services while working together.

The Internet of Energy (IoE) is a network infrastructure that integrates energy and data/information. This Internet of Energy concept helps to balance power generation and energy storage capacity with energy demand in real-time via the advanced metering infrastructure (AMI), demand-response, the e-prosumer, the electric vehicle connected to the grid (V2G) as a power consumption and storage medium, and with various distributed power and energy storage devices. In addition, it is possible to actively integrate and manage the distribution of network management.

The IoE will provide a variety of information and connectivity to energy grids among buildings, cars, and cities, and will present a breakthrough methodology for energy distribution and energy storage, plus monitoring of, and communication with, energy grids. And with renewable energy, energy storage devices, smart meters, energy gateways, smart plugs, and home appliances, energy consumers, manufacturers, and utility suppliers can reduce resources, costs, and the number of target devices, and will provide new and powerful tools for control and management. With the IoE, energy can be delivered bi-directionally anytime, anywhere; and energy consumption monitoring can be performed at all levels, from individual devices to regional, national, and global levels. By providing a reliable, flexible, efficient, and economical energy supply network, consumers can connect and use centralized large-scale power plants and distributed small-scale renewable energy sources, such as solar and wind power, in one convergent system.

The International Renewable Energy Agency (IRENA) published its Innovation Landscape Brief (Blockchain) report about using blockchains [3]. Blockchain technology enables the implementation of smart contracts, which are a form of automated contract processing based on shared networks and trusts between stakeholders, contributing to the integration of much of the renewable energy through system automation [4][5][6]. Smart contracts are automatically executed when certain conditions are met for electricity-exchange transactions. Potential benefits can be improved by reducing transaction costs, increasing transparency, enhancing security, automating management systems, and expanding renewable energy use. Applications of blockchain technology in renewable energy include electricity trading, grid management and system operation, renewable energy development financing, renewable energy certification and management, and electric vehicles [Figure 1]. 


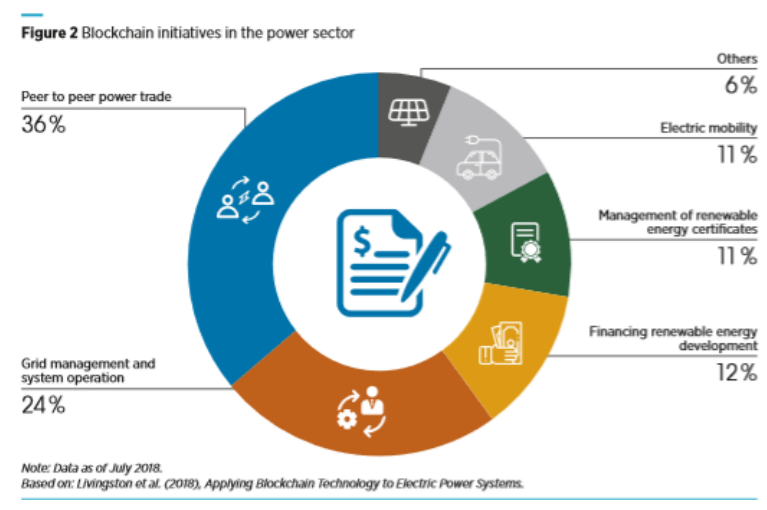

Figure 1. Areas for the application of blockchain in renewable energy [3]

It can be inefficient to have all devices connected to IoT computing. It is desirable to designate a reasonable range of network coverage and to build one fog computing scheme for a group of simple devices within range. In other words, it is efficient to improve the existing gateway into a smarter base device and to maintain the simple and inexpensive low-power and low operation specifications in the remaining connected devices. However, communication costs can be very high if sensors simply transmit the collected data without judgment.

In this paper, we design an energy blockchain transaction architecture that combines blockchain and the energy IoT in a virtual power plant environment. When a consumer who wants to purchase energy through the blockchain smart contract function requests a transaction from a prosumer who sells energy, the transaction information is generated as a block and anonymously disclosed to participants and power companies participating in the power transaction. It is verified, and forms a blockchain with the contents of previous transactions.

This paper consists of Section 1, the introduction, Section 2, research related to Section 3 on the blockchain-based fog-enabled energy IoT architecture, Section 4, providing analysis and evaluation, and Section 5, the conclusion.

\section{Related works}

\subsection{Energy Clouds}

Recently, the power sector has been changing from the centralized supply structure of large power plants, such as thermal power, hydro-electric power, and nuclear power. Distributed power generation is expanding due to emissions regulations and the emergence of prosumers. In addition, the installation price of a distributed power supply is lower and cheaper than existing power generation sources. This change is being developed as a distributed power supply in combination with technologies like cloud computing; technologies such as energy storage, energy efficiency improvement, and demand response are developing into an energy cloud that controls the power grid [Figure 2]. 


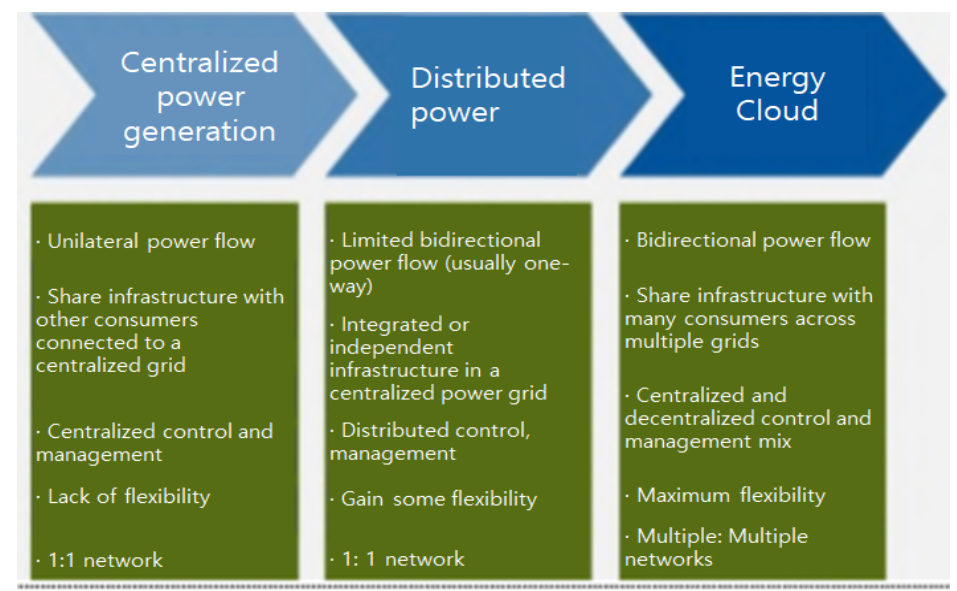

Figure 2. Progress from centralized power generation to the energy cloud

Energy clouds offer economies of scale, flexibility, and resilience. The energy cloud transformation increases distributed power sources, generates and sells electricity on its own, and promotes the growth of the smart grid market.

The energy prosumers are consumers who produce, store, and consume electricity by connecting various distributed power sources, such as photovoltaic cells, fuel cells, and ESSs; they are both consumer and supplier. The producer is an integrated concept [7]. Furthermore, the surplus power remaining after one's consumption can be extended to self-consumption through peer-to-peer transactions in the community. A system for the energy prosumer is a small solar power system or fuel cell used as the main power source, and it is composed of a gateway (inverter, distribution panel, etc.) that connects the ESS for energy storage and controls the whole system [7].

\subsection{Virtual power plant}

Virtual power plants can be used to solve or mitigate issues such as grid disruptions, peak demand, and peak-hour energy costs. On the end-user side, there is a need to strengthen the distribution aspect before building a VPP. This will be of great help in terms of better connectivity and improved reliability.

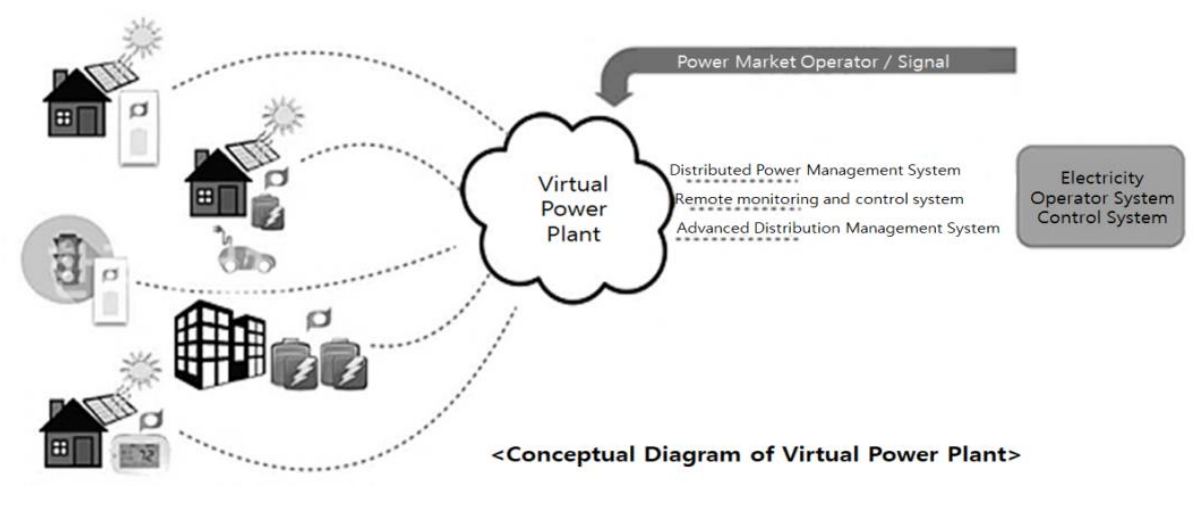

Figure 3. Conceptual diagram of virtual power plants [8] 
Distributed energy resources and the distributed energy resource management solution (DERMS) are key VPP components. The most common DER solutions are solar production and storage solutions. Currently, the most important project related to the VPP is being developed and is progressing based on solar storage technology [8][9].

A VPP is a demand management solution, as shown in Figure 3. It works by integrating multiple end-user power sources and connecting them to a power network. The solution will help to meet demand targets by turning the power generated by the buildings at peak load times into building demand. This demand management pattern can extend to communities as well as buildings.

\section{Blockchain-based Fog-Enabled energy IoT architecture}

In this paper, we propose a fog-connected energy IoT architecture based on a blockchain energy trading platform. [Figure 4] shows the architecture of the fog-connected energy IoT, which is divided into a user who generates data, a fog server, a blockchain that controls energy transactions, and an energy cloud that analyzes and processes all transaction data.

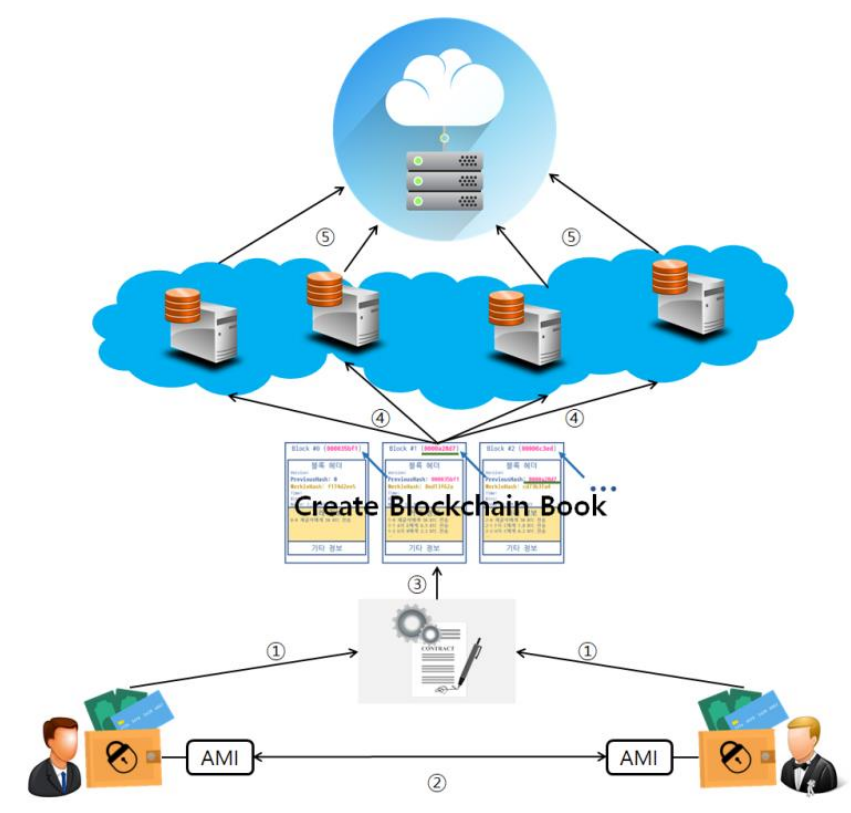

Figure 4. Fog-Enabled energy IoT architecture

The system structure is as follows.

Each user executes a smart contract after receiving data access authority through the fog server with an account (wallet).

A book is created by blocking the transaction of the contract.

The generated books are stored in each fog server, and each fog server also stores the activity $\log$ in the buffer.

Under the smart contract, AMIs trade power.

All the activity logs stored in the fog servers are stored in the energy cloud for analysis and processing. 


\section{Analysis and evaluation}

Data and energy transactions through the blockchain can meet security and integrity. In particular, in order to escape the risk of focusing on sensitive data such as AMI data on cloud servers, we used fog-linked blockchain.

Public transaction ledgers with all information encrypted have a distributed ledger for everyone, so it is impossible to falsify the transaction details to help secure transactions. The user's privacy is protected through the structure of operating the smart contract after the user has been granted data access through the fog as an account (wallet).

We compared and analyzed this paper with [9] and [10] in terms of transparency, safety and efficiency [Table 1]. is an existing centralized IoT system, and [9] is a system that combines IoT with blockchain. In terms of transparency, the three systems [9][10], and this paper all meet transparency. In terms of safety [10], due to the centralized nature, if a centralized server is exposed to an attack, all data may be lost or forged or forged. [9] and this paper use the blockchain system, so all distributed ledgers are held by participants to satisfy the safety of data. In a hyper-connected environment in which many devices are connected, a structure in which traffic is concentrated in one cloud of [9] and [10] is inefficient. The system proposed in this paper is efficient by distributing traffic by adding Fog layer and reducing unnecessary traffic.

Table 1. Comparative analysis of previous studies and this paper

\begin{tabular}{|c|c|c|c|}
\hline Division & {$[10] \mathrm{IoT}$} & [9]BIoT & This paper \\
\hline Transparency & $\circ$ & $\circ$ & $\circ$ \\
\hline Safety & $\times$ & $\circ$ & $\circ$ \\
\hline Efficiency & $\times$ & $\times$ & $\circ$ \\
\hline
\end{tabular}

\section{Conclusion}

In this paper, we designed an energy blockchain transaction architecture that combines a blockchain and the energy IoT. The architecture of the system combines fog computing with an energy blockchain to overcome the limitations of IoT processing speed and storage space. In addition, to secure and automate energy transactions, smart contracts are used by combining the blockchain with the fog layer. The proposed system also stores log activity that controls users in the fog layer between users. The fog layer forwards data to the energy cloud to record and analyze activity logs as well as user-transaction control. Receiving log data, the energy cloud can analyze and process them and extend the data to the potential business model. We compared the proposed architecture with previous studies. In the future, we plan to strengthen security by controlling the user's real-time access and transaction behavior using artificial intelligence in the fog layer.

\section{Acknowledgement}

This research was supported by the Basic Science Research Program through the National Research Foundation of Korea (NRF) funded by the Ministry of Education (2019R1F1A1056507). 


\section{References}

[1] Information and Communication Technology Promotion Center, "Trend of energy research technology on the IoT," Weekly Technology Trends, no.1805, (2017)

[2] Kim Shin Ah and Park Jong-Il, "Energy prosumer business, New opportunities for energy business," LG Instiute of economy, (2016)

[3] IRENA, "Innovation landscape brief: Blockchain," International Renewable Energy Agency, https://www.irena.org//media/Files/IRENA/Agency/Publication/2019/Feb/IRENA_Landscape_Blockchain_(2 019)pdf?la=en\&hash=1BBD2B93837B2B7BF0BAF7A14213B110D457B392, (2019)

[4] YH Seo, JH Hwan, and YI Kong, "Blockchain technology: Prospect and implications in perspective of industry and society," SPRI Issue Report, (2017)

[5] Tokenpost, "What is the Smart Contract?" https://tokenpost.kr/terms/5447, (2017)

[6] Young-Gon Kim, Keol Heo, Jung-In Choi, and Jae-Woo-Wie, "A study on the accounts balancing time of small distributed power trading platform using block chain network," Korean Society for Energy, Journal of Energy Engineering, vol.27, no.4, pp.86-91, (2018)

[7] Anish jindal, Gagangeet Singh Aujla, and Neeraj Kumar, "Survivor: A blockchain based edge-as-a-service framework for secure energy trading in SDN-enabled vehicle-to-grid environment," Computer Networks, vol.153, pp.36-48, (2019) DOI:10.1016/j.comnet

[8] Zhetao Li, Jiawen Kang, Rong Yu, Qingyong Deng, and Yan Zhang, "Consortium blockchain for secure energy trading in industrial internet of things" in IEEE Transactions on Industrial Informatics, vol.14, no.8, pp.3690-3700, (2018)

[9] Arshdeep Bahga, and Vijay K. Madisetti, "Blockchain platform for industrial internet of things," Journal of Software Engineering and Applications, vol.9, no.10, pp.533-546, (2016) DOI:10.4236/jsea.2016.910036

[10] Statis Research Department, "Internet of Things-number of connected devices worldwide 2015-2025," https://www.statista.com/statistics/471264/iot-number-of-connected-devices-worldwide/, (2016) 
Blockchain-based Fog-Enabled Energy loT Architecture

This page is empty by intention. 